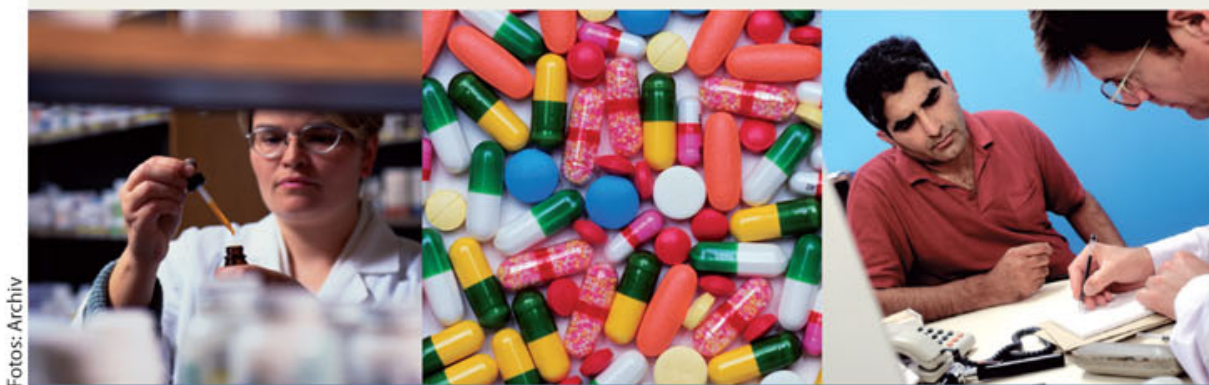

Was ist neu in der medikamentösen Therapie? Wir halten Sie auf dem Laufenden mit Berichten von Kongressen und Symposien der pharmazeutischen Industrie.

Depression, Demenz, ischämischer Schlaganfall

\title{
Was bringt eine Supplementierung mit B-Vitaminen?
}

- B-Vitamine eignen sich möglicherweise, um zerebralen Ischämien und Demenzerkrankungen vorzubeugen. Außerdem könnten sie die Behandlung bei Morbus Parkinson und bei Depressionen unterstützen. Das ist das Ergebnis eines Studien-Reviews von Prof. Dr. Olaf Stanger aus Salzburg und Kollegen.

Folsäure sowie die Vitamine B12 und B6 sind an der Synthese von Neurotransmittern wie Adrenalin, Dopamin und Serotonin beteiligt. Ein Mangel an diesen Vitaminen, wie er besonders bei alten Menschen verbreitet ist, könnte Symptome einer depressiven Verstimmung hervorrufen oder vorhandene Depressionen verstärken. Daher sei die unterstützende Behandlung mit B-Vitaminen womöglich sinnvoll, meinen die Ernährungsexperten aus Österreich, Deutschland und der Schweiz. Nachgewiesen ist bislang, dass Patienten mit Folsäuremangel schlechter und später auf bestimmte Antidepressiva ansprechen als Patienten ohne dieses Defizit.

\section{Ausgleich für medikamentös bedingte Defizite}

Bei Parkinsonpatienten reduziert die LDopa-Behandlung die Spiegel von S-Adenosylmethionin (SAM), einem Methylgruppen-Lieferanten bei der Synthese von Phospholipiden, Hormonen und Neurotransmittern. Die B-Vitamine sind an der Bildung von SAM beteiligt. Zudem hat man bei Parkinsonpatienten beobachtet, dass ein um $60-80 \%$ erhöhter Homocysteinspiegel verstärkt mit dem
Auftreten von Depressionen und zerebralen Ischämien einhergeht. Der erhöhte Bedarf an Folsäure, B6 und B12 sollte deshalb gedeckt werden, so Stanger.

\section{Schlaganfallrisiko sinkt}

Weiter ist hohes Plasma-Homocystein ein Risikofaktor für Atherosklerose. 2,5 mg Folsäure täglich sowie die B-Vitamine verringern einer Metaanalyse zufolge be Risikopatienten mit Gefäßerkrankungen oder Diabetes mellitus das Schlaganfallrisiko um ein Viertel.

Schwedische Wissenschaftler haben bei knapp 1400 Frauen, die 35 Jahre beobachtet worden waren, nachgewiesen, dass bei sehr hohen Homocysteinspiegeln das Risiko für eine Demenzerkrankung um das 1,7-Fache erhöht war, das Risiko für eine Alzheimerdemenz um mehr als das Zweifache. Aus Experimenten war vorher schon bekannt, dass bei hohen Homocysteinkonzentrationen vermehrt amyloidogenes A-beta-Peptid und hyperphosphoryliertes Tau-Protein gebildet werden.

Auch für Antikonvulsiva wie Phenobarbital, Carbamazepin oder Valproat gilt es als gesichert, dass sie den Homocysteinspiegel ansteigen lassen. Bei langfristiger Behandlung mit Antiepileptika empfehlen Stanger und seine Kollegen daher, regelmäßig den Folsäure- und Vitamin-B12-Spiegel zu kontrollieren.

\section{Dr. med. Thomas Meißne}

Quelle: DACH-Liga Homocystein e. V. (www. dach-liga-homocystein.org)

\section{Kurz notiert}

Nutzen der Cholinesterase-Hemmer erneut bestätigt ÿ Für die Acetylcholinesterase-Hemmer (AChEHemmer) ist bei Patienten mit leichter bis mittelschwerer Alzheimerdemenz ein Nutzen in Hinsicht auf die kognitiven Fähigkeiten belegt. Zu diesem Urteil kam das IQWiG bereits in seinem Abschlussbericht zur Nutzenbewertung im Jahr 2007. Zum selben Ergebnis kommt jetzt auch eine vom G-BA geforderte Aktualisierungsrecherche des IQWiG. Im Hinblick auf die Aktivitäten des täglichen Lebens konstatiert das IQWiG zumindest "Hinweise auf einen Nutzen".

Für das weltweit am meisten verordnete Antidementivum, den AChE-Hemmer Donepezil (Aricept ${ }^{\odot}$ ), gibt es umfangreiche Studien, die zusätzlichen zu den positiven Wirkungen auf Kognition, Alltagskompetenz und klinischen Gesamteindruck $u$. a. auch eine verlangsamte Krankheitsprogression und einen reduzierten Pflegeaufwand zeigen.

DPP-4-Hemmer als Zusatztherapie zu Insulin ÿ Die Liste der zugelassenen Indikationen für den DPP-4Hemmer Sitagliptin (Januvia ${ }^{\circ}, X^{-}$ levia ${ }^{\circ}$ bzw. Janumet ${ }^{\circ}$, Velmetia ${ }^{\circ}$ ) ist nochmals länger geworden. Das orale Antidiabetikum kann jetzt mit oder ohne Metformin als Zusatztherapie zu Insulin verordnet werden, wenn Diät und Sport plus eine stabile Insulintherapie den Blutzucker bei Typ-2-Diabetes nicht ausreichend senken können. 